\title{
Design and Construction of an Authorized Car Parking Alert System
}

\author{
Peter Otchere ${ }^{1,2^{*}}$, Wisdom Opare ${ }^{1,2}$ \\ ${ }^{1}$ School of Energy and Power Engineering, Jiangsu University, Zhenjiang 212013, Jiangsu, China \\ ${ }^{2}$ Faculty of Engineering, Electrical and Electronic Engineering Department, Takoradi Technical University, Ghana
}

\begin{abstract}
DOI: $10.36348 /$ sjet.2019.v04i12.006 $\quad$ | Received: 20.12.2019| Accepted: 27.12.2019| Published: 30.12 .2019
*Corresponding author: Peter Otchere
\end{abstract}

\section{Abstract}

In this modern world, with continuous rise of vehicle ownership and usage, parking space has become a big challenge in companies, institutions and major cities as a whole. Research is on-going in this area to help solve this ubiquitous problem by the use of smart parking systems. Currently, extensive use of wireless technology applications to help solve the parking issue shows that digital data dissemination is the way to go. Infra-red (IR) technology which is a Wireless Sensor technology has attracted increased attention over the years due to its enormous application potential in diverse fields and also its potential of been an efficient and cost-effective alternative solution. This paper proposes an Authorized car parking alert system which provides advanced features like remote parking monitoring and parking reservation mechanism. The authorised car parking alert system is a typical internet of things (IoT) application that is developed using IR technology to provide services to users and parking owners of a smart institution and city. The paper describes the overall system design to implementation in the view point of sensor networks. Consideration is made of a smart parking infrastructure where sensors are laid down on the parking spots to detect car presence and IR readers are embedded into parking slot to identify cars and help in the arrangement of the parking area. Devices are endowed with wireless communication capabilities for reporting to the ground where the situation recognition is performed and also prevent unauthorized cars from entering or parking at any unassigned area.

Keywords: Car parking alert system, wireless technology, sensors, infra-red, transmitter, receiver, comparator.

Copyright @ 2019: This is an open-access article distributed under the terms of the Creative Commons Attribution license which permits unrestricted use, distribution, and reproduction in any medium for non-commercial use (NonCommercial, or CC-BY-NC) provided the original author and source are credited.

\section{INTRODUCTION}

Wrong parking is one of the challenges in our societies, cities, institutions, companies, schools and apartments where drivers park anyhow and anywhere they wish to primarily because of space scarcity. However, to build an environment that utilizes smart parking infrastructure, vehicle occupancy detection is of fundamental importance [1]. According to the San Francisco traffic Agency, $30 \%$ of the city traffic is due to search for vacant parking slots. If vehicle users have information about the number of vacant parking slots, a huge amount of unnecessary traffic can be minimized and therefore will make the cities green and efficiently organized. Thus the smart parking system is considered beneficial for both car park operators and car park patrons as well as in terms of environment conservation.

From the literature it is known that there are already some existing methods that have been developed to solve this problem of parking and examples are the Smart parking system [2] which is a typical IoT application that benefit from advances in sensor, actuator and RFID technologies to provide several services to its users and parking owners of a smart city. For the car park operators, the information gathered via the implementation of the Smart Parking System [2] can be exploited to predict future parking patterns. Thus pricing strategies can be manipulated according to the information obtained to increase the company's profit. In terms of environment conservation, the level of pollution can be reduced by decreasing vehicle emission (air pollutant) in the air. This can be attributed to the fact that vehicle travel is reduced. As fuel consumption is directly related to vehicle miles travelled, it will be reduced as well. Patrons also benefit from smart parking system as parking space are able to be fully utilized due to a safer, optimized and more efficient system implemented. The system is made more efficient as vehicle travel time and search time are significantly reduced due to the information provided by the smart parking system. With the information provided, drivers are able to avoid car parks that are fully occupied and locate vacant parking spaces with ease elsewhere. The number of vehicles parked illegally by the roadside which leads to traffic 
congestion is also reduced as it is absorbed into the car parks. Most importantly, traffic congestion is reduced and all these eventually lead to convenience for everyone.

Multi story car parking system [3] is another method used for parking and retrieving of cars with the use of a pallet and lifts system. The intention is to compact more cars by reducing the space needed to park the same number of cars in the same space. Car parks can be situated on ground level, above or below ground level or a combination of both three making the system efficient and thus a space saving one. This design is normally developed using an 8051 microcontroller. The Multi-storeyed car parking systems [3] help to minimize the parking area as we find ourselves in a modern world where space has become a very big challenge. It also enables the parking of vehicles, floor after floor and thereby reducing the space used. Multi-story car parks provide lower building cost per parking slot, as they typically require less building volume and less ground area than a conventional facility with the same capacity. They also offer greatest possible flexibility for the realization of optimum parking solution by applying time-saving vertical and horizontal movements that take place simultaneously ensuring fast parking and retrieval times of cars.

There is also the Smart card parking system [4]. Smart card parking system is well established in the parking market, with vendors providing solution for all segments: single space meters, multi-space meters, and off-street parking. The large parking vendors have installed closed-loop contact smart card solution in cities and the parking operator issues the smart cards, manages retail outlet, manages cardholder queries, reloads the card and manages the entire card system. Many of the cities implementing smart cards are doing so by leveraging their existing meter infrastructure, replacing single-space meters with smart card enabled single-space meters. Other are upgrading their singlespace meters to accept smart cards adding additional multi-space where appropriate. In those cities, the multi-space, single space meters share the same smart card program, and multi-space anywhere in the city can be used to load value unto the smart card. Some of the solution only works with one parks payment vendor's technology, although there are instances of collaboration between non competing vendors in some cities. These meters accept smart cards and typically accept coins. The Smart card technology [4] is another innovative method that allows customers to use one card for parking and for other payments, search as transit, tolls or small purchases. Using smart cards can increase the efficiency of parking operation in several areas including security, labour requirement and equipment.
The problem of detecting the vacancies in parking space is well investigated in the literature, where the proposed solutions are mostly computer vision or sensor-based systems [5]. These sensor-based devices are built of particular sensor(s) that sense the presence, while the sampling logic and data delivery through the radio front-end is controlled by a microcontroller (MCU) unit. These are already available in the market as commercial solutions, and vendors guarantee 5-10 years without the need of changing the batteries. Figures 1 and 2 show typical types of these solutions and conversely with the Authorized car parking alert system it also helps to save space at the parking area. In the modern world where parking space has become a very big problem, it has become very important to save parking spaces especially where there are a whole lot of cars that need to be parked. This authorized car parking system enables the parking of vehicles, slot after slot, and thus reduces the space used. It also prevents unauthorized cars from entering or parking at any unassigned area. Furthermore, it reduces the level of manpower-skills as it defeats the assignment of an instructor or security to drive away cars at assigned parking slots. Based on the programmed requirement, any number of cars can be parked accordingly. This makes the system an efficient and space-saving one. This novel design is developed using IR technology.

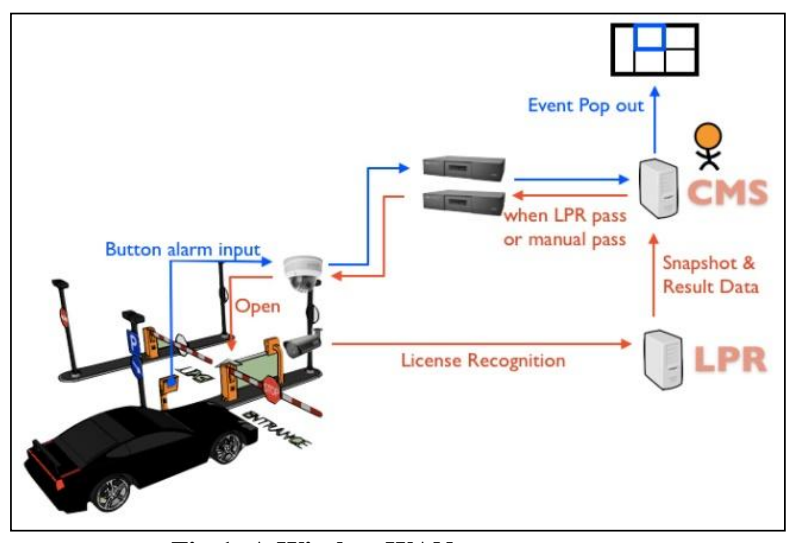

Fig-1: A Wireless WAN sensor system

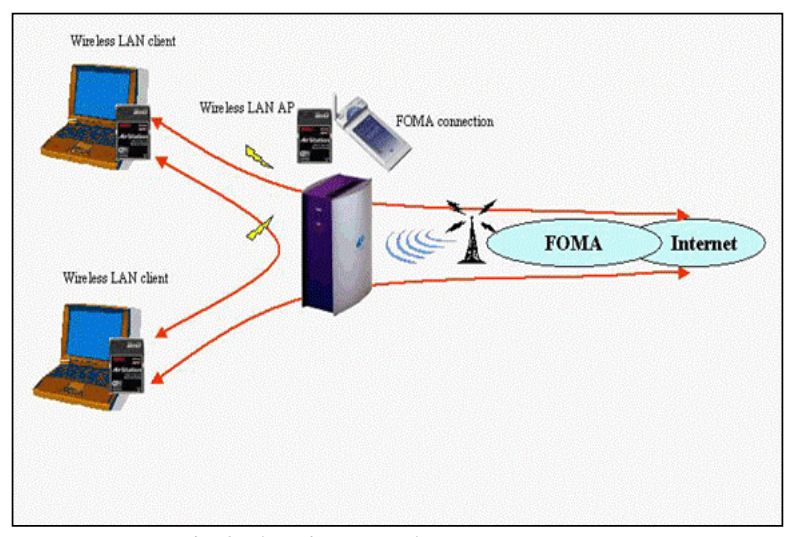

Fig-2: A Wireless LAN sensor system 
Smart parking environments can use both passive and active infrared sensors. Passive infrared sensors detect heat that is radiating from surrounding objects, such as human objects passing the parking space $[5,6]$. For example, when a driver parks and gets out of the vehicle, the sensor will trigger its presence. Such passive infrared sensor devices are usually used in combination with other vehicle detection sensors. On the other hand, active infrared sensor devices measure the distance to any object placed in front of them [7, 8]. A disadvantage is the sensitivity to the daylight, and therefore is more appropriate for usage in the indoor environment. On the other hand, ultrasonic sensor devices can be used to calculate the distance between obstacles and are more appropriate for outdoor environments. Ultrasonic sensor devices depend on the round-trip time of an ultrasound that is reflected on the nearby object. For precise measurement and better performances, these could be further improved if the ultrasonic sensor distance values are enhanced with the equation that involves the temperature and humidity sensing values [9, 10]. Further, accelerometers that measure the ground vibrations can be used to detect whether a vehicle is approaching a parking spot. In the combination with other sensors, such as optical sensor devices that detect variations in light level, a better estimation of car being present in a parking space could be provided. In such scenario, sensors are installed in a space where car presence creates an obstacle [11, 12]. However, optical sensors still depend on the light source and their accuracy. One of the most practical and common sensor used today on parking spots is the magnetometer sensor which measures the current magnetic field and detects a metal object within its presence [13]. Cameras also find its space in the detection of vehicles in parking areas $[14,15]$. Cameras used for smart parking require a more complex and real-time image processing used to detect the occupancy. Although such devices usually have been found to violate users privacy (for example, detection of car registration), they find applications in criminal investigations. Other sensor devices may also be used for car detection, such as piezoelectric sensor device and inductive loops [16]. However, these sensor devices are usually installed on road surface and detect if vehicle is passing or not. RFID find its application in smart parking systems, usually in payment solution systems $[17,18]$. Once the sensors are installed on the parking slots, they need to deliver information about the occupancy. There are many communication methods to convey information to the centralized system, usually via wireless communication, either short or long range. These technologies are as follows: Bluetooth/BLE, Wi$\mathrm{Fi}$ and ZigBee/Z-Wave/DigiMesh, or long- range, like Sigfox, LoRa, Ingenu and NB-IoT/LTE-M. Long range communication benefit from single hop communication to the base station, while short range technologies such as ZigBee sometimes require the presence of a multihop relay mechanism until they reach the city infrastructure.
As discussed earlier, it can be seen that to create a smart parking system, usually a combination of sensors is employed to increase the accuracy of the complete sensing mechanism. For purposes of this study, and to further increase the reliability, a low cost IR sensor device is considered as an orthogonal sensing logic approach. The spectrum of invisible electromagnetic waves with the wavelength between $700 \mathrm{~nm}$ to $1 \mathrm{~mm}$ is called infrared. IR is a well-known technology that provides wireless data transmission through wireless sensors [8]. The IR detectors are divided to different categories based on their wavelength, detectability, sensitivity, method of detection, sensing time, and used technology thus PIR (Passive IR Resistors) or PID (Passive IR Motion Detectors). They are called "passive" because of their functionality in collecting the IR values of an environment without radiation. In fact, they play the role of a broker between optics and electronics. They are used for object detection, distance measurement, human sensing, motion detection, and proximity sensing. A lot of implementations in robotics, security systems, and factories can be observed all over the world. The night and thermal vision are some other abilities that they have. The PIR thermometers (without touching) and flame monitoring systems are designed and used in different industries as well. They are also exploited for analysis of gas and moisture. The remote control of electronic home appliances and automatic door opening systems observed in daily life of people mostly use IR. The first idea of detecting the IR waves was offered in 1940 and has evolved during the years [19]. The mercury cadmium telluride ( $\mathrm{HgCdTe})$ is an alloy of cadmium telluride (CdTe) and mercury telluride $(\mathrm{HgTe})$ proposed after several efforts for finding the best material for detecting in 1959 [20]. The $\mathrm{HgCdTe}$ is most widely used presently and its advantage in detecting infrared compared to the others is proved [21]. Current phase of research is focused on multi-element detectors and arrays for imaging [22]. The infrared sensors are divided into two types: "Photo voltaic" that produces current and "Photo conductor" that changes its resistance according to the received photon with figures 3 and 4 showing samples of these sensors.

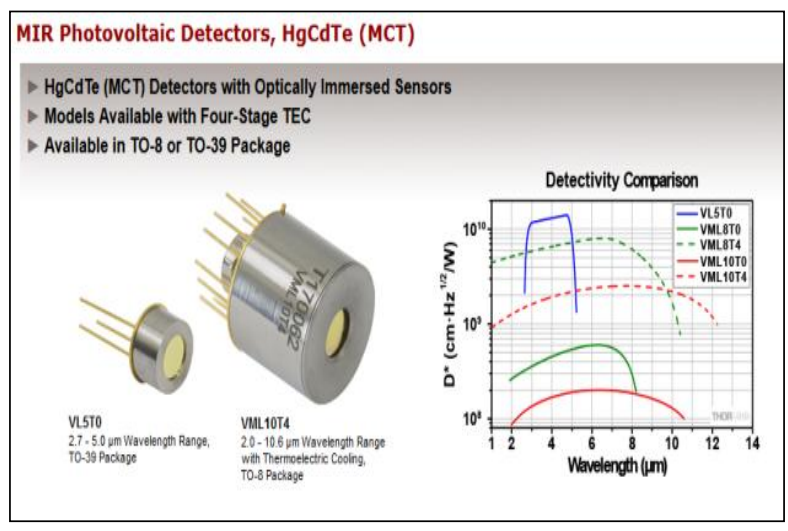

Fig-3: A Photovoltaic sensor 
Peter Otchere \& Wisdom Opare., Saudi J Eng Technol, Dec. 2019; 4(12): 522-534

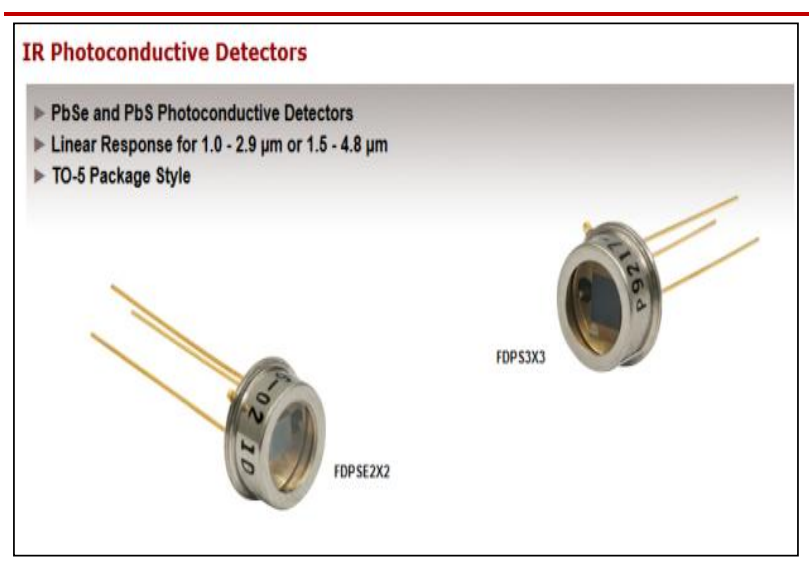

Fig-4: A Photoconductor sensor

Authorized car parking alert application uses IR technology and requires no human intervention when used for vehicle detection. This technology helps the drivers to quickly check-in and checkout from the parking lot and also makes the parking space secure. The IR sensor at the entry point helps in avoiding multi check-ins thus avoiding traffic congestion in the parking lot. The IR technology enables automatic Parking fee collection thus saving time of the drivers. The main components of IR technology that are used for the purposes are IR labels, IR readers, software system and a barrier to control the gate. The transaction management, reporting and operational tasks are controlled by the software used in IR. A database management system is also used to manage and record the vehicle tracking data and is considered within software requirements.

This paper gives an overview of used technologies for detecting the vacancy in the parking slots. Moreover, it analyses the modes of operation of popular sensors used nowadays and the information provided in this paper can be used for better understanding the potential of using such sensors. Based on information from existing literatures and knowledge in how IR sensor works, this study established a novel design system of a device that will authorize cars or vehicles to enter into a car parking area, prevent unauthorized vehicles from entering into unassigned parking area, provide sufficient parking area, reduce the usage of "no parking sign notices", reduce disarrangement of vehicles at parking fields and then constructed the prototype of the artefact.

\section{MATERIALS AND METHODS}

Based on existing technologies [2-4] on the research of parking systems this paper proposes a new device, an authorized car parking alert system, that is a smart parking infrastructure which is suitable and more efficient. Authorized car parking alert system is simply an alert system with sensor to identify an individual user assigned to a particular parking area in companies, institutions and metropolises. There is a trigger of an alarm or a signal is given to the security check point with the help of a speaker when an unauthorized (intruder) person parks within the reserved areas. With the authorized car parking alert system sensors are laid down on the parking spots to detect car presence, RFID readers are embedded into parking gate to identify cars and also help in the billing of the smart parking. Basically this system is made of a Sensing Layer that defines a platform where sensor devices are embedded into the parking lot to detect car presence. Three types of sensor devices are used namely; Slave Devices, Master devices and Anchor. The devices are endowed with wireless communication capabilities for reporting to a gateway where the situation recognition is performed. The sensor devices are tasked to play one of these roles:

- Slave sensor node device also called "receivers", are placed on the parking spots to detect presence/absence of vehicles.

- Master sensor node devices also called "transmitters", are placed at one of the edges of a parking lot to detect presence and collect the sensor readings from their connected slave node devices and transmit these readings to a gateway for further processing. The slave devices are connected to the master devices through wired communication using the I2Cprotocol.

- Relay sensor nodes, also called anchor/repeat node devices, are used as repeaters to increase the wireless sensor coverage of the parking lot for efficient routing of the readings. They are located strategically at specific locations in the parking lot to increase the coverage and connectivity of the wireless sensor network. For sensing purposes, micro-controllers equipped with ultrasonic sensors are used, and an ARM-based Panda board was used as the gateway. While slave and master nodes are placed based on geographic constraints, the optimal placement of the relay/anchor sensor nodes is an important parameter upon which the efficiency of the parking system depends. The optimal placement of sensor in smart parking is formulated as an integer linear.

As compared to the existing parking devices the authorized car parking alert system has an added feature of giving authorization to vehicles to park at a designated parking area where they are assigned to park and also prevent unauthorized vehicles from parking at a parking spot where they are not assigned to park. Authorized car parking alert system make parking easy and convenient. Furthermore, it works more efficiently and it is also not complex to install. Possible benefits also include increasing revenue, improving accessibility, utilization of assets, reducing congestion and improving safety [23].

Programming is done based on multi-objective problem optimization whereby the sensor network engineering efficiency in terms of coverage and lifetime maximization, as well as its economic gain in terms of 
the number of sensor deployed for a specific coverage and lifetime are taken into consideration. An exact solution to the node placement problem using singlestep and two-steps solution implemented in the most language based on the Xpress-MP suit of libraries is proposed. Experimental results reveal the relative efficiency of the single-step compared to the two-steps model on different performance parameters. These results are consolidated by simulation result, which reveal that the solution out forms a random placement in terms of energy consumption, delay and throughput achieved by a smart parking network. The device is also reliable because its design configuration is such that it works twenty four hours all day and night. The designed proximity is also within 2inches away from the device and the approaching vehicle.

\section{Description of design components}

The various components used for the design and construction of the artefact are briefly described.

\section{Phototransistor (sensor) BTX 81}

The Phototransistor (sensor) BTX 81 is an optic-electronic component that receives and convert infrared signal into electrical signal [24]. These phototransistors were used to receive transmitted infrared signal, adjust selected sensitivity range and did not require additional amplification in their operation. The Phototransistors though can be used in two modes; ACTIVE and SWITCH modes, was used in the switch mode thus the phototransistor was either off or on as per digital logic response to the object sensed.

\section{Infrared LED (Sensor) BTW 34}

The IR LED, also known as IR transmitter, is a special purpose LED that transmits infrared rays in the range of $760 \mathrm{~nm}$ wavelength [25] and was used in this study. They, along with IR receivers, are commonly used as sensors. The appearance is same as a common LED. Since the human eye cannot see the infrared radiations, it is not possible for a person to identify whether the IR LED is working or not, unlike a common LED. But to verify its function ability, the camera on a cell phone was used because it can show the IR rays being emanated from the IR LED in a circuit.

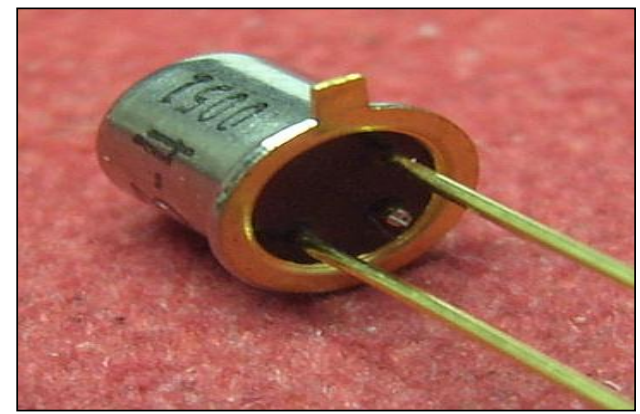

Fig-5: Infrared Phototransistor (sensor)

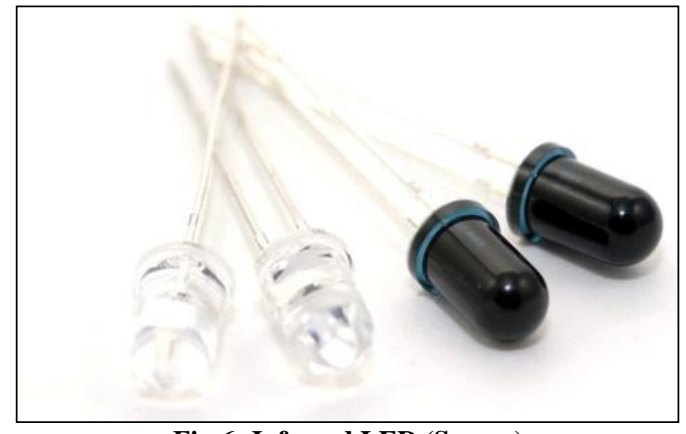

Fig-6: Infrared LED (Sensor)

\section{Bipolar Transistor PNP BC554}

A bipolar junction transistor (bipolar transistor or BJT) which is a type of transistor that uses both electron and hole charge carriers [26] was used in the study to amplify current. In contrast, unipolar transistors, such as field-effect transistors, only use one kind of charge carrier. For their operation, BJTs use two junctions between two semiconductor types, n-type and p-type. BJTs are manufactured in two types, NPN and PNP, and are available as individual components, or fabricated in integrated circuits, often in large numbers to be used as amplifiers or switches, giving them wide applicability in electronic equipment, including computers, televisions, mobile phones, audio amplifiers, industrial control, and radio transmitters.

\section{Electrolytic Capacitor}

Electrolytic capacitors (e-caps) are polarized capacitors whose anode (+) is made of a particular metal on which an insulating oxide layer forms by iodization, acting as the dielectric of the electrolytic capacitor. An electrolyte that covers the surface of the oxide layer in principle serves as the second electrode (cathode) (-) of the capacitor. Due to their very thin dielectric oxide layer and enlarged anode surface, electrolytic capacitors based on the volume have a much higher capacitance - voltage (CV) product compared to ceramic capacitors or film capacitors, but a much smaller $\mathrm{CV}$ value than electrochemical super capacitors [27]. The Electrolytic capacitor types $10 u \mathrm{~F}$ $16 \mathrm{~V}$ and $470 \mathrm{yF} 25 \mathrm{~V}$ were used in the study.

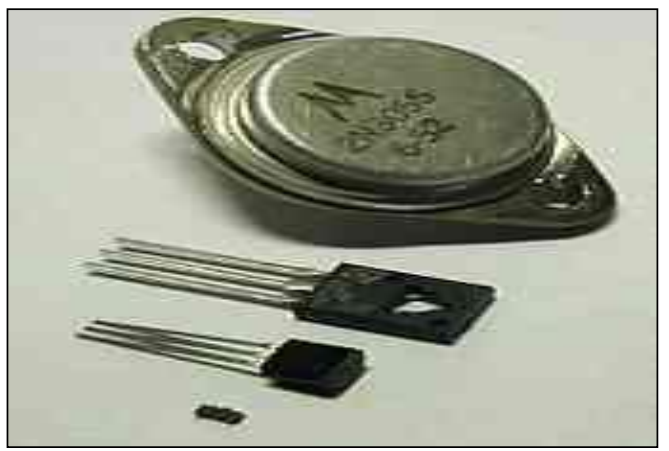

Fig-7: Bipolar Transistor PNP BC554 


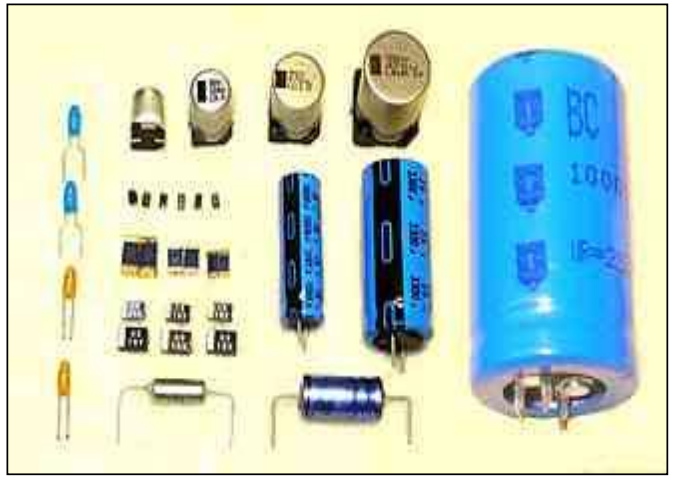

Fig-8: Electrolytic Capacitor

\section{Mica Capacitor 102}

Mica is a group of natural minerals and mica capacitors are capacitors that use mica as the dielectric. There are two types of mica capacitors: clamped mica capacitors and silver mica capacitors. Clamped mica capacitors are currently considered obsolete due to their inferior characteristics and hence silver mica capacitors are used instead [28]. They are made by sandwiching mica sheets coated with metal on both sides. This assembly is then encased in epoxy in order to protect it from the environment. Mica capacitors are generally used when the design calls for stable, reliable capacitors of relatively small values. They are low-loss capacitors, which allow them to be used at high frequencies, and their value does not change much over time. Mica minerals are very stable electrically, chemically and mechanically. Because of its specific crystalline structure binding, it has a typical layered structure. This makes it possible to manufacture thin sheets in the order of $0.025-0.125 \mathrm{~mm}$. The most commonly used are muscovite and phlogopite mica. The first has better electrical properties, while the second has a higher temperature resistance. Mica is delved in India, Central Africa and South America. The high variation in raw material composition leads to high cost needed for inspection and sorting. Mica doesn't react with most acids, water, oil and solvents [29].

\section{Buzzer}

For good performance, general purpose piezo buzzer is commonly used in alerting/alarming circuits [30] thus a PCB mountable piezo buzzer that can be soldered easily to a PCB board is used for the construction. It also has long life, stable performance, of high quality with small outline transistor (SOT) plastic package.

\section{Rectifier Diode IN4007}

The most popular application of the diode is for rectification purposes. Simply defined, rectification is the conversion of alternating current (AC) to direct current (DC). This involves a device that only allows one-way flow of electrons and this is exactly what a semiconductor diode does. The simplest kind of rectifier circuit is the half- wave rectifier. It only allows one half of an AC waveform to pass through to the load [31].

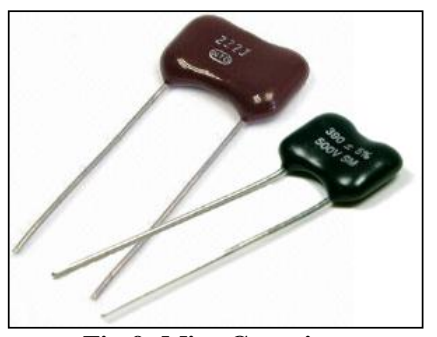

Fig-9: Mica Capacitor

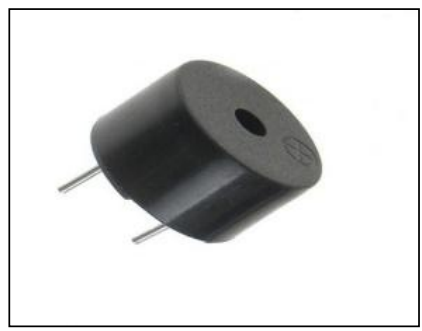

Fig-10: Piezo Buzzer

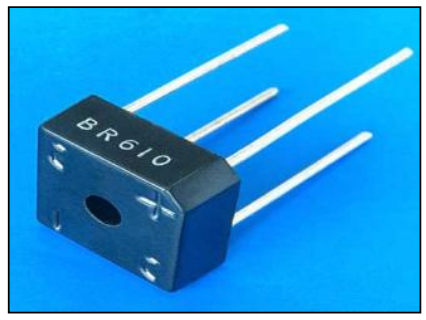

Fig-11: Rectifier Diode

\section{Resistors R2 to R5 $100 \Omega$}

The resistors used are passive two-terminal electrical component that implements electrical resistance as a circuit element. Resistors are generally used to reduce current flow, and, at the same time, may act to lower voltage levels within circuits. In electronic circuits, resistors are used to limit current flow, to adjust signal levels, bias active elements, and terminate transmission lines among other uses. High-power resistors, that can dissipate many watts of electrical power as heat, may be used as part of motor controls, in power distribution systems, or as test loads for generators [32]. Fixed resistors have resistances that only change slightly with temperature, time or operating voltage. Variable resistors can be used to adjust circuit elements (such as a volume control or a lamp dimmer), or as sensing device for heat, light, humidity, force, or chemical activity. Resistors are common elements of electrical networks and electronic circuits and are ubiquitous in electronic equipment. Practical resistors as discrete components can be composed of various compounds and forms. Resistors are also implemented within circuits. The electrical function of a resistor is specified by its resistance thus common commercial resistors are manufactured over a range of more than nine orders of magnitude. Therefore the nominal value of the resistance falls within a manufacturing tolerance. 
Relay

A reed relay was used as a reed switch enclosed in a solenoid. The switch has a set of contacts inside an evacuated or inert gas-filled glass tube which protects the contacts against atmospheric corrosion; the contacts are made of magnetic material that makes them move under the influence of the field of the enclosing solenoid or an external magnet. Reed relays can switch faster than larger relays and require very little power from the control circuit. However, they have relatively low switching current and voltage ratings. Though rare, the reeds can become magnetized over time, which makes them stick 'on' even when no current is present. Changing the orientation of the reeds with respect to the solenoid's magnetic field can however resolve this problem [33].

\section{Jump wire}

Jump wires that are short electrical wires with solid tips at each end (or sometimes without them, simply "tinned"), were used to interconnect the components in a breadboard. In other instances, they are used to transfer electrical signals from anywhere on the breadboard to the input/output pins of the microcontroller [34]. Jump wires are fitted by inserting their "end connectors" into the slots provided in the breadboard that has beneath its surface a few sets of parallel plates that connect the slots in groups of rows or columns depending on the area. The "end connectors" are inserted into the breadboard in the particular slots that need to be connected in the specific prototype without soldering.

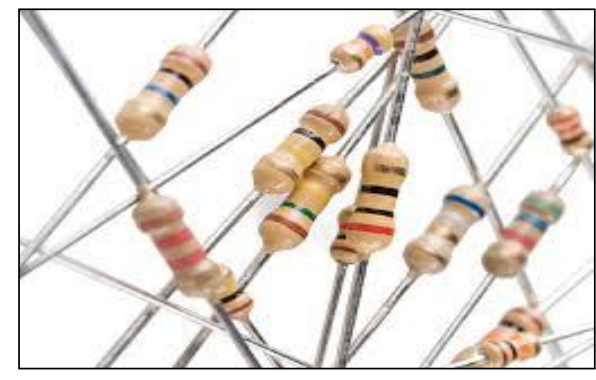

Fig-12: Resistors

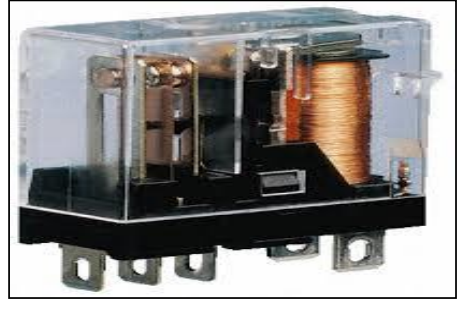

Fig-13: Relay

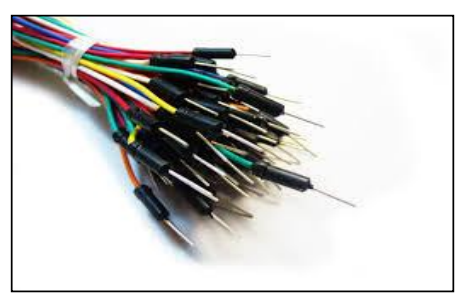

Fig-14: Jump wires

\section{Design of Prototype Model}

The system operates under a 9v d.c and 3v d.c supplies, infrared receivers, frequency generators (transmitters), frequency comparators and a buzzer. The infrared frequency generator (1) is supplied by the $3 \mathrm{v}$ power supply and the other systems are supplied with the $9 \mathrm{v}$ source. The infrared frequency generator(1) sends infrared frequency to the infrared receiver(1), the infrared receiver(1) receives the infrared signal and convert it into electrical signals for the comparator(1) to compare the infrared converted signal and deliver it to the buzzer deactivator to deactivate the buzzer from beeping when the car is authorized. On the other hand when an unauthorised car approaches, the infrared frequency generator(2) sends infrared frequency to the infrared receiver(2), the infrared receiver(2) receives the infrared signal and convert it into electrical signals for the comparator(2) to compare the infrared converted signal and deliver it to the buzzer activator to activate the buzzer to beep.

The Power Side of the circuit is made up of battery, relay and capacitor. The IR Receiver (1) is made up of Phototransistor and Infrared LED, the IR Transmitter (1) is made up of IR diode, resistor and battery, the IR Transmitter (2) is made up of resistor, fixed capacitor and IR diode/sensor, and the IR receiver (2) is made up of IR sensor/diode, resistor and capacitor.

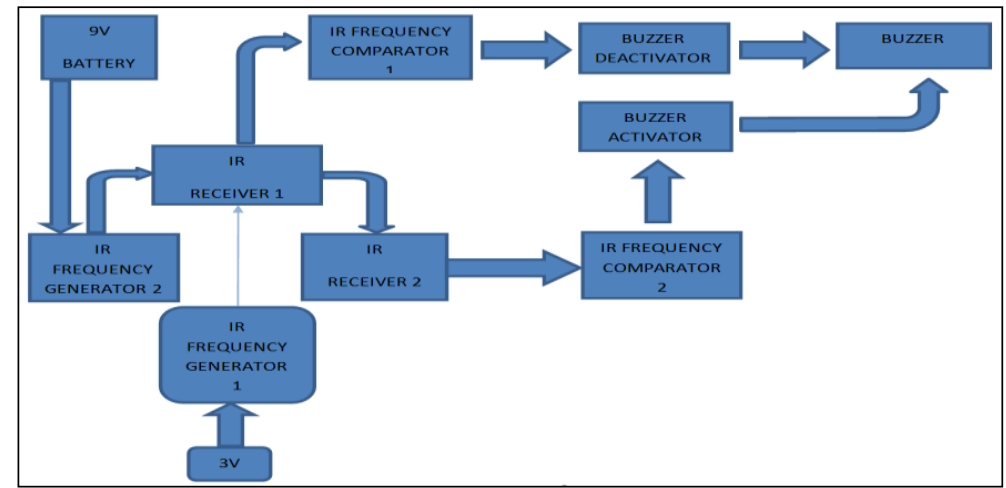

Fig-15: Block diagram of Design concept 


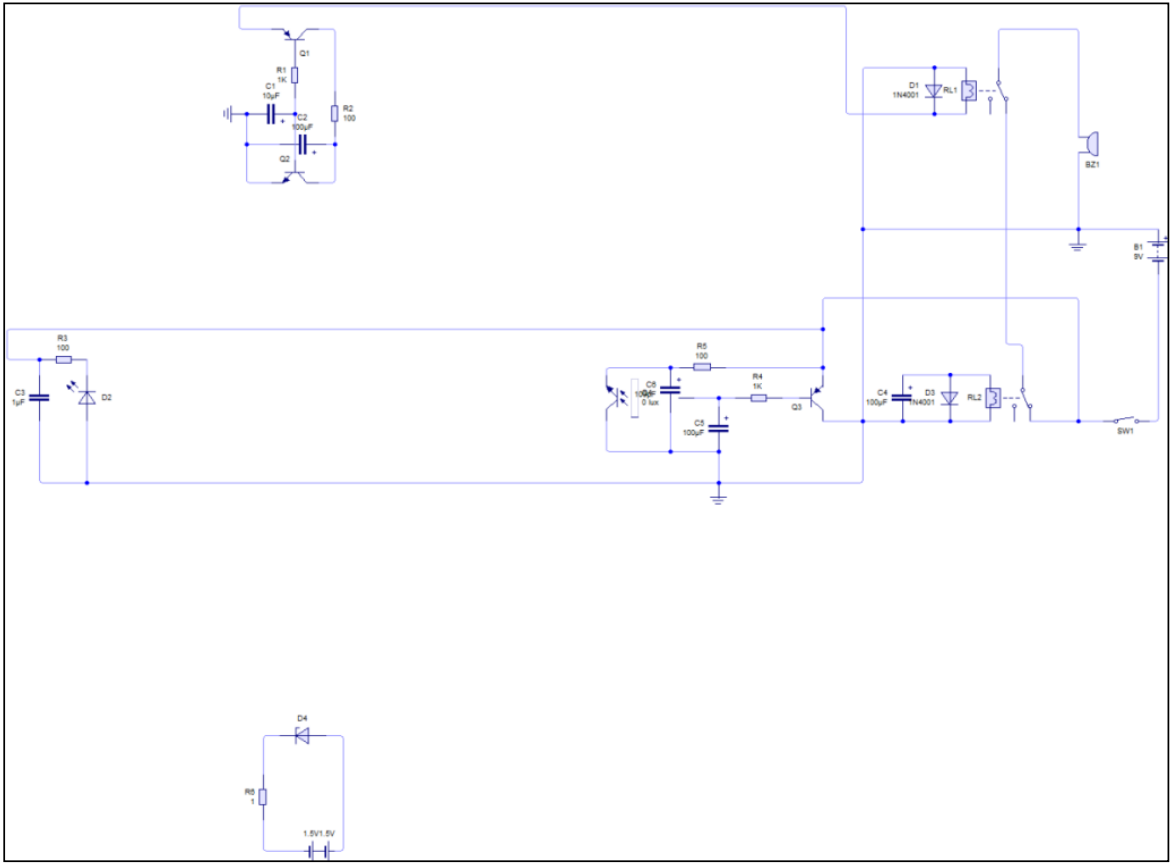

Fig-16: Circuit Design

\section{Screen shot of simulated circuit diagram}

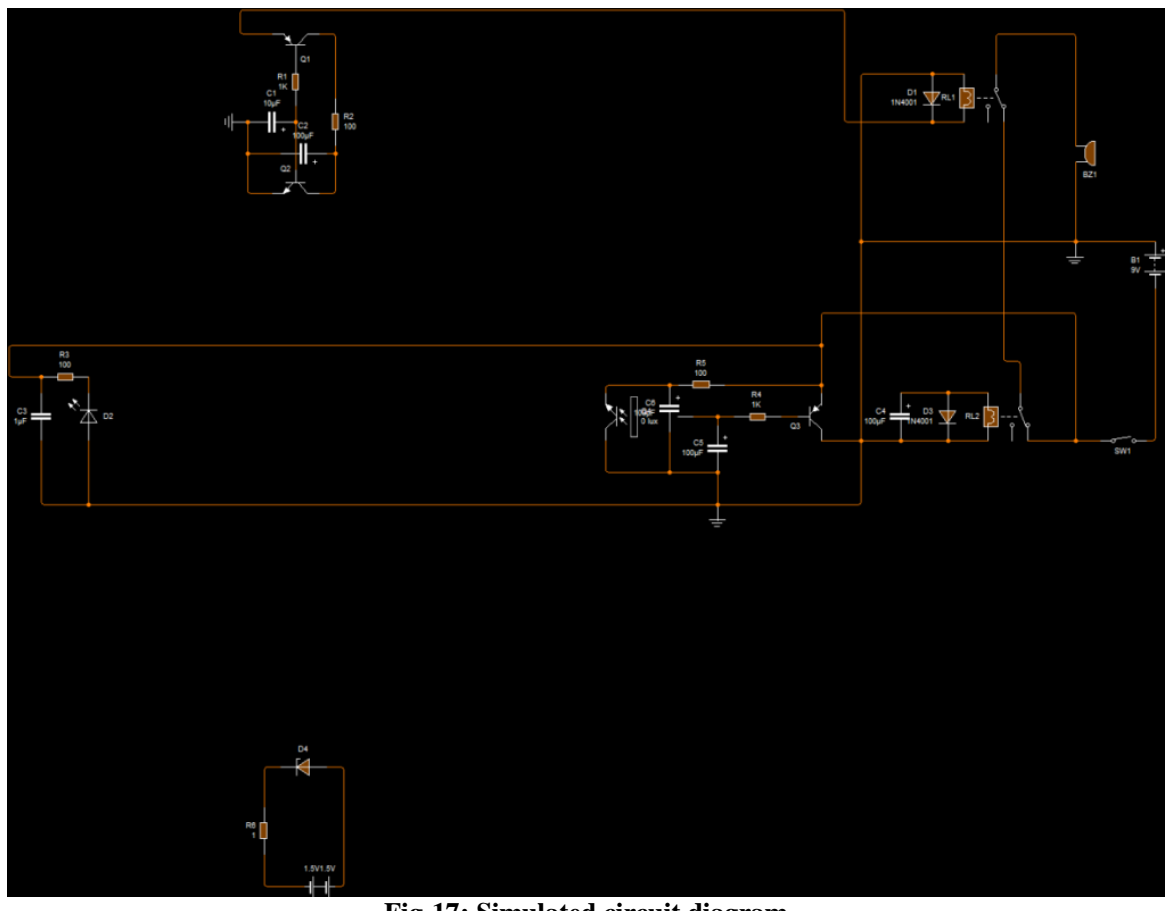

Fig-17: Simulated circuit diagram

\section{Component specification}

The following are the materials and component specifications used in the project 
Peter Otchere \& Wisdom Opare., Saudi J Eng Technol, Dec. 2019; 4(12): 522-534

Table-1: Component specification

\begin{tabular}{|c|c|c|}
\hline Quantity & Component & Specification \\
\hline 1 & Phototransistor (Sensor) & BTX 81 \\
\hline 1 & Infrared LED (Sensor) & BTW 34 \\
\hline 2 & Bipolar Transistor & BC554 \\
\hline 5 & Electrolytic Capacitor & 10чF 16V, 470 чF 25V \\
\hline 1 & Mica Capacitor & 102 \\
\hline 1 & Buzzer & $9 \mathrm{~V}$ \\
\hline 2 & Rectifier Diode & IN4007 \\
\hline 6 & Resistors & R2 to R5 100 $\Omega$ \\
\hline 1 & Switch & ON/OFF \\
\hline 2 & Relay & $9 \mathrm{~V}$ \\
\hline 15 & Jumper Cables & $0.5 \mathrm{~m}$ \\
\hline 2 & Battery & $9 \mathrm{~V}$ \\
\hline 1 & Zener Diode & $3 \mathrm{~V}$ \\
\hline
\end{tabular}

Component analysis and testing was done before they were used for the construction purpose of the "Authorized car parking alert system". After testing each component, each component was able to withstand the testing and was suitable to be used in this project which even made the design and construction more portable. The procedure used in the testing and analysis of each component is as follows;

\section{Resistors}

Visual inspection was made on the resistors to make sure there was no blackening or char. The resistor values were read visually as the resistors value are printed on the resistors with values of the smaller resistors indicated by colour coded bands. The digital multi-meter (DMM) was prepared to measure the resistor values for confirmation ensuring that the DMM comes on and does not indicate a low battery condition. The adjustable scale of the DMM was set to the next higher value than the expected resistors value and measured the resistance by connecting the two legs of the resistor to the DMM leads. Resistors have no polarities, so it does not matter which DMM lead is connected to which resistor leg. After determining the actual resistance of the resistors, the resistors that give an accurate reading were selected and those that measured outside of the acceptable value range were replaced.

\section{Capacitors}

The capacitors were visually inspected to see if there were no leaks, cracks, bulges or other signs of deterioration evident otherwise the capacitor was replaced. The DMM knob was turned to the capacitance measurement mode and tested the capacitors to ensure that the correct capacitor was used in the circuit. In the testing, the test leads of the DMM were connected to the capacitor terminals for a few seconds to allow the multi-meter to automatically select the proper range. The measurement displayed signified whether the capacitance values are within the measurement range.

\section{Diode}

The ohms range on the DMM was selected and then connected the cathode terminal of the diode to the terminal marked positive on the multi-meter, and the anode to the negative or the common terminal. The DMM knob was turned to read ohms and a low reading was obtained. The connection was then reversed and this time a higher resistance reading was obtained indicating that the diode was in good condition.

\section{NPN Transistor/PNP Transistor}

The ohms range on the DMM was selected and then the base terminal of the transistor was connected to the terminal marked positive (usually coloured red) on the multi-meter. The terminal marked negative or common (usually marked black) was connected to the collector and the resistance measured. It read open circuit as the meter deflected for a PNP transistor. The terminal marked positive, was maintained still connected to the base, with the positive terminal this time connected to the emitter and repeated the measurement. The reading was again open circuit as the meter deflected for PNP transistor.

Now the connection was reversed with the base of the transistor, this time connected to the negative or common (black) terminal of the test meter and connected the terminal marked positive, first to the collector and measured the resistance. Then connected it to the emitter and also measured the resistance. In both cases, the meter deflected indicating open circuit for a PNP transistor.

Next the meter negative or common was connected to the collector and the meter positive to the emitter. The measurement by the meter read open circuit thus the meter read open circuit for both PNP and NPN transistor types. The connection was reversed so that the meter negative or common was connected to the emitter and meter positive to the collector. We then checked again whether the meter read open circuit and thus confirmed that the transistors had passed the entire test and realised that they were basically functioning and all the junctions were intact. 
Infrared LED Sensor

When testing the infrared sensor, it was found out that there is a semi-conductor/chip inside the sensor, and was powered with $5 \mathrm{v}$ before functioning.

\section{Relay}

The relay schematic or data sheet was consulted and a basic visual inspection of the relay was done. The coil requirement of the relay was determined and it was found out that the control coil was diode protected. The contact configuration of the relay was also accessed as testing was done on the deenergised/energised conditions of the relay as well as checking of the energised condition of the contacts. A multi-meter in diode test mode was used to confirm the findings.

\section{Precautionary measures taken in the design and construction}

The following were the precautionary measures taken when working with the component;

- Personal protective equipment (PPE) was worn when soldering or cutting wires or component leads.

- Proper ventilation was ensured when soldering or using cleaning compounds.

- Hands were washed with detergents after handling solder, even lead free solder since lead free solders may be even more poisonous than leaded solder.
- Power source was detached from any piece of equipment before working on it.

\section{Limitation of the design}

- The smart device battery has to be in good working condition always

- The sensing devices must be free from any obstacles

\section{RESULTS AND DISCUSSION}

The system is designed to operate under a $9 v$ d.c and $3 \mathrm{v}$ d.c supplies. The infrared transmitter 1 is supplied by the $3 \mathrm{v}$ supply and the other systems are supplied with the $9 \mathrm{v}$ source. The system component comprises of infrared receivers, frequency generators, infrared receivers, infrared comparators and a buzzer. As previously discussed, the $9 \mathrm{v}$ battery supplies the circuit with a D.C. power source constantly and the IR transmitter generates and transmit IR signal to the IR receiver system. The IR Receiver system receives the infrared signal transmitted by the IR transmitter and converts to electrical signal for the IR frequency comparator to compare the converted signal. The comparators determine the output of the system, whether the buzzer will sound or not. At normal condition, the infrared frequency generator (2) generates and transmits infrared frequency to the receiver (2). This receiver converts the signal to the comparator to compare the state of the infrared receiver.

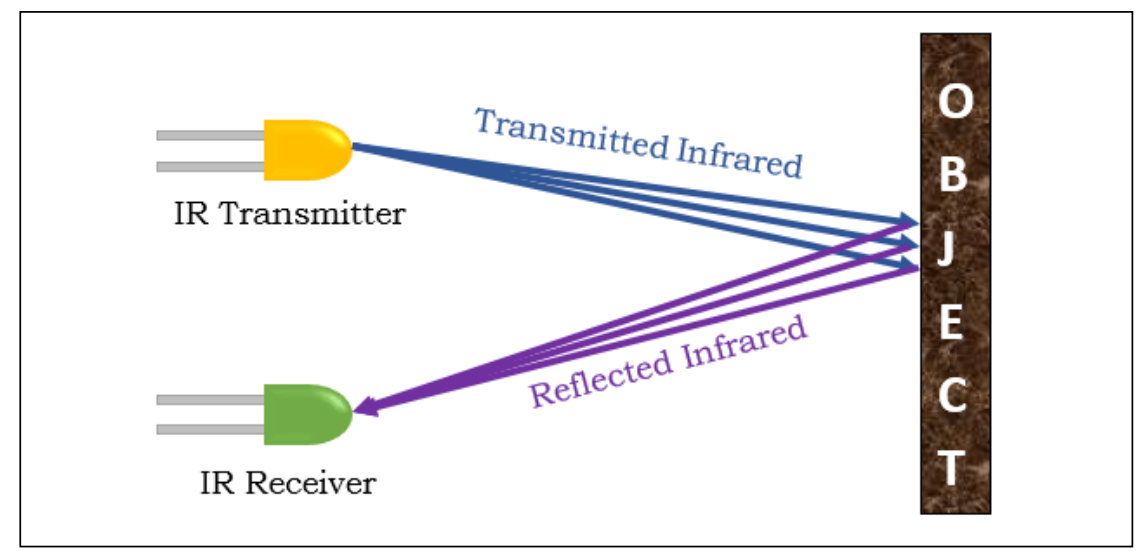

Fig-18 Pictorial diagram of IR Transmitter and IR Receiver

For an unauthorized car, the buzzer elevates because the transmitted frequency is blocked from reaching the infrared receiver (2), the output of the infrared receiver gets into a passive state and the comparator sends a signal to the buzzer to sound. This state shows that there is the presence of an unauthorized car. For an authorized car, the $3 \mathrm{~V}$ battery supplies the IR transmitter with a D.C. power source that enables the IR transmitter to generate IR frequency in the authorized car that consecutively communicates to the IR receiver. When an authorized car which has an infrared frequency generator in it, approaches the secured area, the infrared frequency generator transmit an infrared frequency for the infrared receiver (1) to receive. The IR Receiver after receiving the transmitted infrared signal by the IR transmitter then converts the signal into electrical signal and then sends the signal to the IR frequency comparator that compares the received infrared signal transmitted and then switches on an electromagnetic relay. This relay deactivates comparator (2) thus the buzzer deactivator switches the state of the buzzer from high to low in the case of an authorized car therefore, when the infrared is blocked from reaching the infrared receiver (2) the comparator (2) is in a passive state, and the buzzer stays in an off state hence an output sound does not come from the buzzer in the form of an alarm. Figure 18 shows the pictorial 
of the IR transmitter and IR Receiver and figure 19 parameters of the authorized car parking alert system. shows the block diagram of the input and output

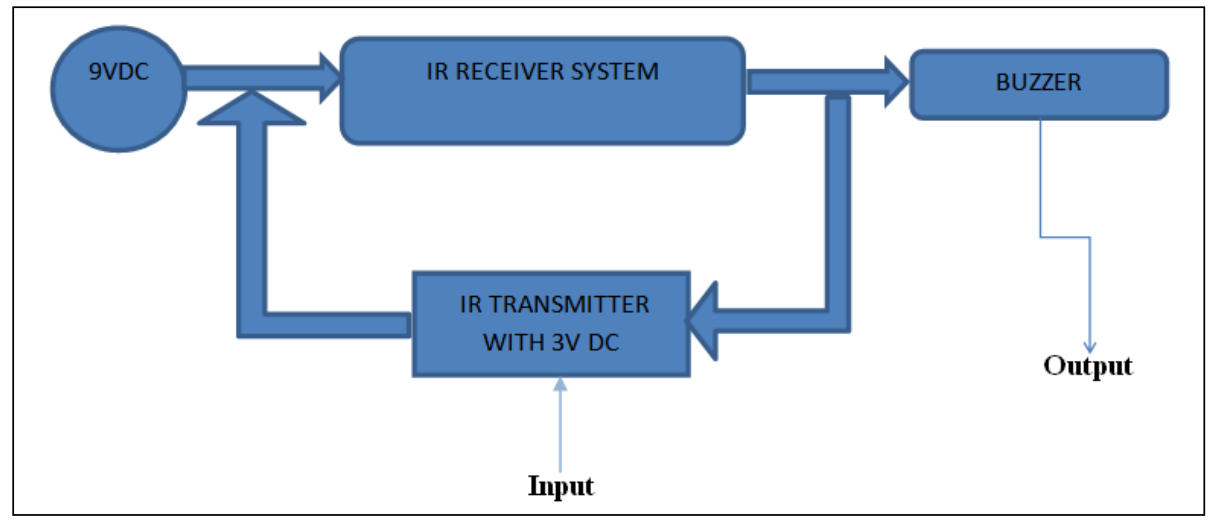

Fig-19: Block diagram of Input and output parameters of authorized car park alert system

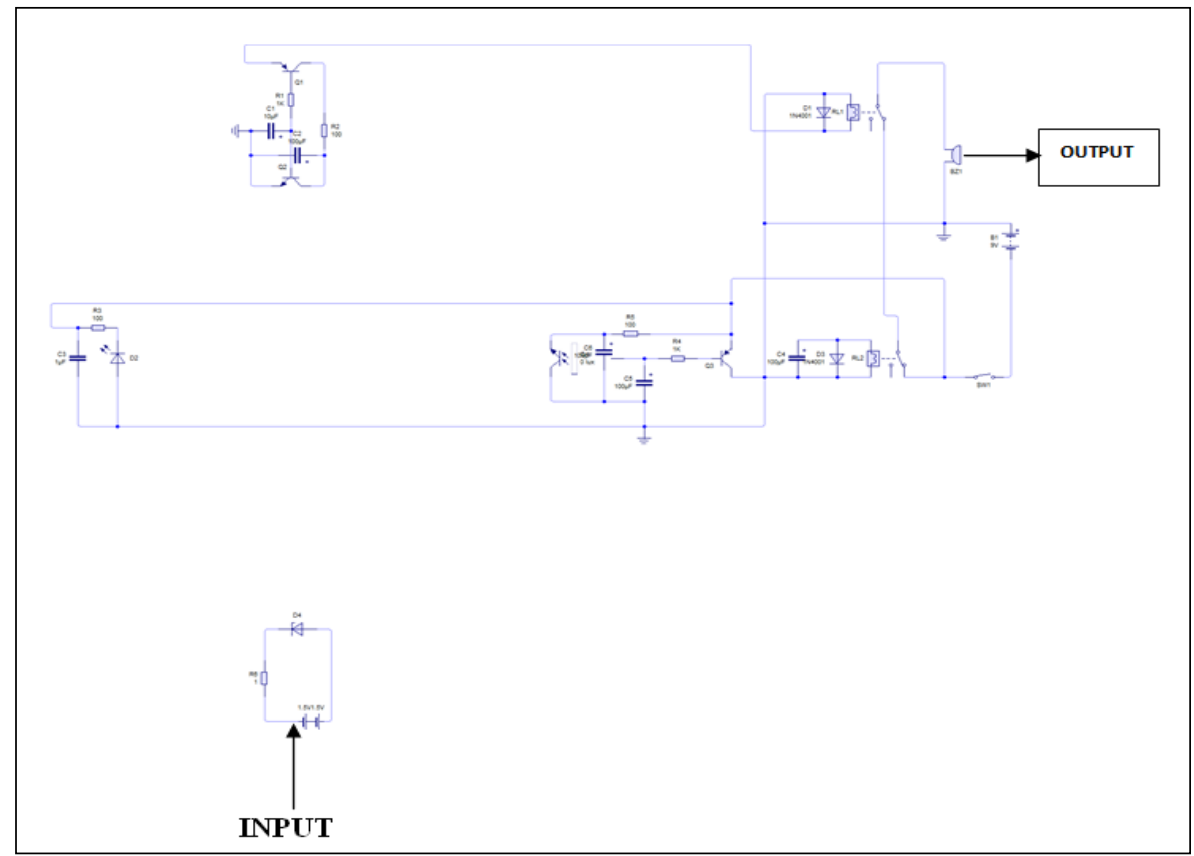

Fig-20 Circuit diagram of Input and output parameters of authorized car park alert system

After attaining certainty that all the components were in good condition to be used, the construction of the prototype "authorized car parking alert system" artefact commenced and the design was implemented on a pc board as per the circuit diagram. A thick card board material was used to design the housing package for the parking area and prototype toy cars with blue and white colours were used to represent both the authorized and unauthorized vehicles respectively. After the design had been constructed, the artefact was tested and its performance worked as anticipated. Preliminary test results show that the performance of this "Authorized car parking alert system" can effectively satisfy the needs and requirements of existing parking hassles.

From fig. 21a it shows that there are three parking slots and the blue car is parked at the authorized slot while the white car is parked at the unauthorized slot. The middle parking slot as indicated by fig. $21 \mathrm{~b}$ is its authorized parking slot so the buzzer sounded for the white car at the instance displayed by fig. 21a. When the white car parked at the middle slot as indicated by fig. $21 \mathrm{c}$ there was no buzzer sound thereby indicating that the construction of the designed artefact worked accordingly. 


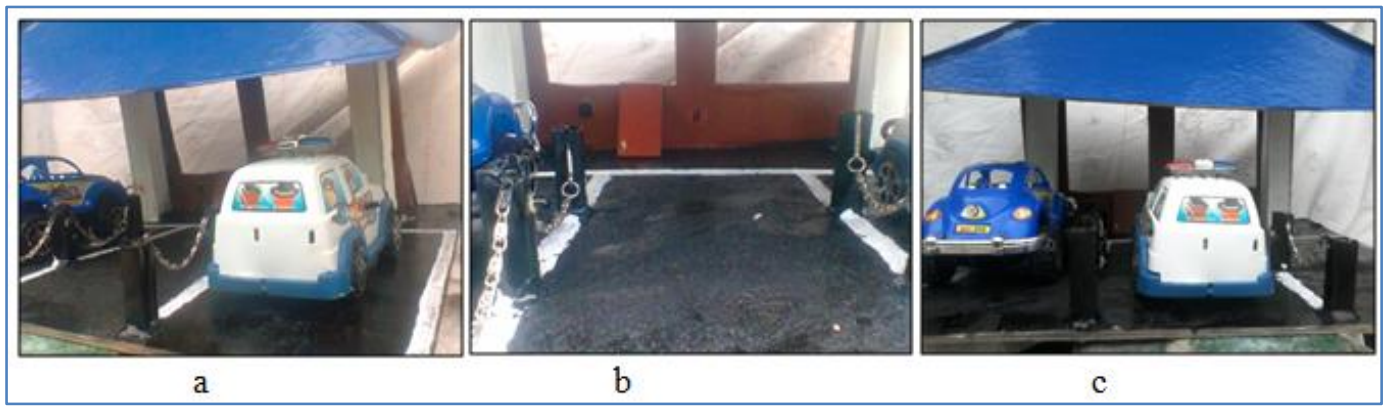

Fig-21: Prototype of authorized car park alert system

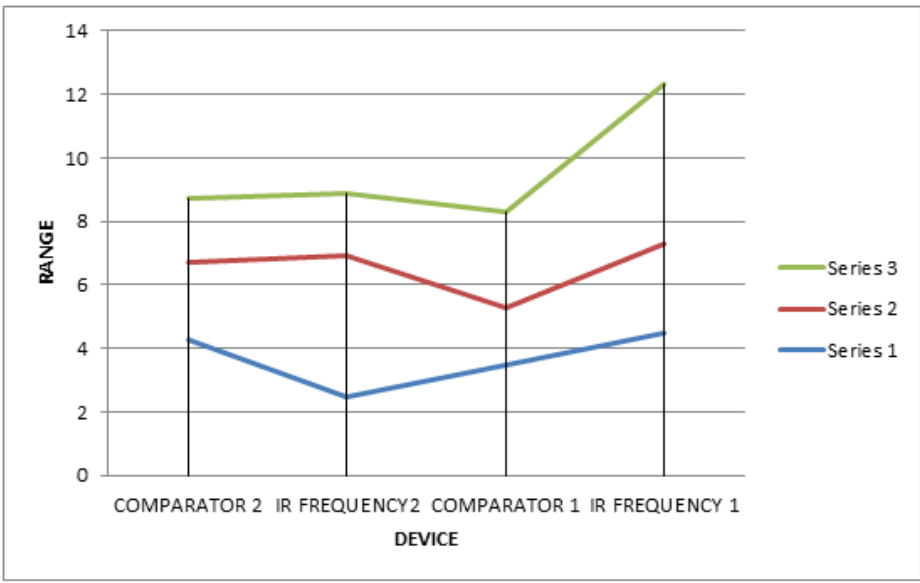

Fig-21: Performance curves/graphs of authorized car park alert system

\section{CONCLUSION}

The section summarizes the major findings of the study and draws conclusions from the findings. In this modern world, parking space has become a big challenge in companies, institutions and cities as a whole, where mostly, people park at a parking spot where they are not permitted to park. The "authorized car parking alert system" is to help eliminate these existing problems, whereby the system simply identifies an individual user assigned or unassigned within a particular parking area in companies, institutions and metropolises. The system triggers an alarm or gives a signal to the security check point with the help of a buzzer when any unauthorized (intruder) person parks within the reserved areas. In conclusion, authorized car parking alert system as a solution will address this challenge as it brings onboard an improved and professional way of parking.

\section{REFERENCES}

1. Gu, J., Zhang, Z., Yu, F., \& Liu, Q. (2012). Design and implementation of a street parking system using wireless sensor networks. In Proceedings of the 10th IEEE International Conference on Industrial Informatics (INDIN), Beijing, China, 25-27 July, 1212-1217.

2. Li, X., \& Ranga, U.K. (2009). Design and implementation of a digital parking lot management system. Technol. Interface J., 10.

3. Jian, M.S., Yang, K. S., \& Lee, C.L. (2008). Modular RFID parking management system based on existed gate system integration. WSEAS Trans. Syst., 7, 706-716.

4. Pala, Z., \& Inanc, N. (2007, September). Smart parking applications using RFID technology. In 2007 1st Annual RFID Eurasia (pp. 1-3). IEEE.

5. Lin, T., Rivano, H., \& Le Mouël, F. (2017). A survey of smart parking solutions. IEEE Transactions on Intelligent Transportation Systems, 18(12), 3229-3253.

6. Larisis, N., Perlepes, L., Kikiras, P., \& Stamoulis, G. (2012). "U-park: Parking management system based on wireless sensor network technology," in Intl Conf on Sensor Technologies and Applications.

7. Moguel, E., Preciado, M., \& Preciado, J. (2014). "Smart parking campus: An example of integrating different parking sensing solutions into a single scalable system," in ERCIM News Smart Cities.

8. Urdiain, L., Romero, C., Doggen, J., Dams, T., \& Van Houtven, P. (2012). "Wireless sensor network protocol for smart parking application experimental study on the arduino platform," in Intl Conf on Ambient Computing, Applications, Services and Technologies,

9. Kianpisheh, A., Mustaffa, N., Limtrairut, P., \& Keikhosrokiani, P. (2012). Smart parking system (SPS) architecture using ultrasonic detector. International Journal of Software Engineering and Its Applications, 6(3), 55-58.

10. Coric, V., \& Gruteser, M. (2013, May). Crowdsensing maps of on-street parking spaces. In 2013 IEEE International Conference on 
Distributed Computing in Sensor Systems (pp. 115122). IEEE.

11. Jeffrey, J., Patil, R., Narahari, S., Didagi, Y., Bapat, J., \& Das, D. (2012). "Smart parking system using wireless sensor networks," in Intl Conf on Sensor Technologies and Applications.

12. Chinrungrueng, J., Sunantachaikul, U., \& Triamlumlerd, S. (2007). "Smart parking: An application of optical wireless sensor network," in Intl Symp on Applications and the Internet Wksp.

13. Srikanth, S. V., Pramod, P. J., Dileep, K. P., Tapas, S., \& Patil, M. U. (2009, May). Design and implementation of a prototype smart parking (spark) system using wireless sensor networks. In 2009 International Conference on Advanced Information Networking and Applications Workshops (pp. 401-406). IEEE.

14. Sevillano, X., Màrmol, E., \& Fernandez-Arguedas, V. (2014, July). Towards smart traffic management systems: Vacant on-street parking spot detection based on video analytics. In 17th International Conference on Information Fusion (FUSION) (pp. 1-8). IEEE.

15. Thai-Nghe, N., \& Chi-Ngon, N. (2014, December). An approach for building an intelligent parking support system. In Proceedings of the Fifth Symposium on Information and Communication Technology (pp. 192-201). ACM.

16. Yan, G., Yang, W., Rawat, D. B., \& Olariu, S. (2011). SmartParking: A secure and intelligent parking system. IEEE Intelligent Transportation Systems Magazine, 3(1), 18-30.

17. Bagula, A., Castelli, L., \& Zennaro, M. (2015). On the design of smart parking networks in the smart cities: An optimal sensor placement model. Sensors, 15(7), 15443-15467.

18. Djenouri, D., Karbab, E., Boulkaboul, S., \& Bagula, A. (2015). Networked wireless sensors, active rfid, and handheld devices for modern car park management: Wsn, rfid, and mob devs for car park management. International Journal of Handheld Computing Research (IJHCR), 6(3), 3345 .

19. Rogalski, A. (2010). Infrared detectors. CRC press.

20. Lawson, W. D., Nielsen, S., Putley, E. H., \& Young, A. S. (1959). Preparation and properties of HgTe and mixed crystals of HgTe-CdTe. Journal of Physics and Chemistry of Solids, 9(3-4), 325329.

21. Norton, P. (2002). HgCdTe infrared detectors. Optoelectronics review, (3), 159-174.

22. Rogalski, A. (2011). Recent progress in infrared detector technologies. Infrared Physics \& Technology, 54(3), 136-154.

23. Yass, A. A., Yasin, N. M., Zaidan, B. B., \& Zeiden, A. A. (2011, June). New design for intelligent parking system using the principles of management information system and image detection system.
In Proceedings of the 2009 International Conference on Computer Engineering and Applications, Manila, Philippines (Vol. 68, p. 360364).

24. Bong, D.B.L., Ting, K.C., \& Lai, K.C. (2008). Integrated approach in the design of car park occupancy information system (COINS). IAENG Int. J. Comput. Sci., 35, 7-14.

25. Tang, V.W.S., Zheng, Y., \& Cao, J. (2006). An intelligent car park management system based on wireless sensor networks. In Proceedings of the 2006 1st International Symposium on Pervasive Computing and Applications, Urumqi, China, 3-5 August 2006, 65-70.

26. Bi, Y., Sun, L., Zhu, H., Yan, T., \& Luo, Z. (2006). A parking management system based on wireless sensor network. Acta Automatica Sinica, 32(6), 968.

27. Benson, J. P., O'Donovan, T., O'Sullivan, P., Roedig, U., Sreenan, C., Barton, J., ... \& O'Flynn, B. (2006, November). Car-park management using wireless sensor networks. In Proceedings. 2006 31st IEEE Conference on Local Computer Networks (pp. 588-595). IEEE.

28. Geng, Y., \& Cassandras, C.G. (2012). A new 'smart parking' system infrastructure and implementation. Procedia-Soc. Behav. Sci., 54, 1278-1287.

29. Lu, R., Lin, X., Zhu, H., \& Shen, X. (2009, April). SPARK: A new VANET-based smart parking scheme for large parking lots. In IEEE INFOCOM 2009 (pp. 1413-1421). IEEE.

30. Samaras, I., Evangeliou, N., Arvanitopoulos, A., Gialelis, J., Koubias, S., \& Tzes, A. (2013). KATHODIGOS-a novel smart parking system based on wireless sensor network. In Intelligent Transportation Systems (Vol. 1, pp. 140-145). Transp. Res. Board.

31. Chinrungrueng, J., Dumnin, S., \& Pongthornseri, R. (2011, August). IParking: A parking management framework. In 2011 11th International Conference on ITS Telecommunications (pp. 63-68). IEEE.

32. Gu, J., Zhang, Z., Yu, F., \& Liu, Q. (2012, July). Design and implementation of a street parking system using wireless sensor networks. In IEEE 10th International Conference on Industrial Informatics (pp. 1212-1217). IEEE.

33. Nawaz, S., Efstratiou, C., \& Mascolo, C. (2013, September). Parksense: A smartphone based sensing system for on-street parking. In Proceedings of the 19th annual international conference on Mobile computing \& networking (pp. 75-86). ACM.

34. Rose, R. (2001). A smart technique for determining base-station locations in an urban environment. IEEE Transactions on Vehicular Technology, 50(1), 43-47. 